03

\title{
Структурные характеристики газожидкостного течения в микроканале с Т-образным смесителем
}

\author{
(C) В.В. Кузнецов ${ }^{1,2}$, И.А. Козулин ${ }^{1,2}$ \\ ${ }^{1}$ Институт теплофизики им. С.С. Кутателадзе СО РАН, Новосибирск \\ ${ }^{2}$ Новосибирский государственный университет \\ E-mail: vladkuz@itp.nsc.ru
}

Поступило в Редакцию 22 ноября 2016 г.

Представлены результаты экспериментального исследования структурных характеристик течения смеси азот-вода в горизонтальном микроканале с Т-образным входом. Эксперименты проведены в канале прямоугольного сечения $250 \times 315 \mu \mathrm{m}$ в условиях доминирующего влияния капиллярных сил. Структурные характеристики течения установлены с использованием двухлучевого лазерного сканирования и скоростной видеосъемки на расстоянии 500 калибров от входа в широком диапазоне приведенных скоростей газа и жидкости. Предложен новый метод идентификации режима течения на основе статистической обработки данных лазерного сканирования и построена карта режимов течения.

DOI: 10.21883/PJTF.2017.21.45162.16574

Разработка и внедрение микротехнологий в различных областях техники привели к созданию широкого спектра микрожидкостных систем с поперечным размером элементов в диапазоне от 10 до $300 \mu \mathrm{m}$, предназначенных для работы с малыми объемами жидкостей и газов [1]. Двухфазные микрожидкостные системы получили широкое распространение во многих приложениях, на их основе созданы МЭМС-устройства (МЭМС - микроэлектромеханические системы), микроканальные двухфазные теплообменники, топливные процессоры и топливные элементы водородной энергетики. Обзор режимов двухфазных газожидкостных течений в микроканалах представлен в [2]. Течение смеси азот-вода в микроканалах квадратного и круглого сечений с гидравлическим диаметром $D_{h} \cong 100 \mu \mathrm{m}$ и прямоугольных микроканалах рассмотрено в $[3,4]$. В работе [5] представлены режимы газожидкостного течения в микроканале с зазором $300 \mu \mathrm{m}$ и шириной 
от 10 до $30 \mathrm{~mm}$. Сравнение режимов течения смесей азот-вода и азот-этанол приведено в [6] для микроканалов прямоугольного сечения с $D_{h}=187.5-218 \mu \mathrm{m}$ и Т-образным входом. В [7] рассмотрено влияние статического давления на режим течения смеси азот-вода в микроканале с сечением $300 \times 600 \mu \mathrm{m} \mathrm{c} \mathrm{Т-образным} \mathrm{входом.} \mathrm{В} \mathrm{этих}$ работах выделено шесть основных режимов газожидкостного течения: пузырьковый, снарядный, снарядно-кольцевой, кольцевой с кольцеобразными волнами, кольцевой и вспененный. Границы режимов течения зависят от формы каналов и степени влияния капиллярных сил, характеризуемых величиной чисел Бонда Во $=g\left(\rho_{\text {liq }}-\rho_{g a s}\right) D_{h}^{2} / \sigma$ и Вебера $\mathrm{We}=\rho_{L}\left(J_{g a s}+J_{l i q}\right)^{2} D_{h} / \sigma$. Здесь $J-$ приведенная скорость, определенная как отношение объемного расхода к сечению канала, $\sigma$ - поверхностное натяжение, $\rho$ - плотность, $g$ - ускорение силы тяжести, индексы liq и gas соответствуют жидкости и газу. В большинстве исследований использованы микроканалы кругового сечения, в то время как каналы микрожидкостных систем обычно имеют прямоугольную форму с Т- или Ү-образной конфигурацией входов для жидкости и газа.

Из анализа опубликованной литературы можно заключить, что основным методом определения режима течения в микроканале являются наблюдения с помощью скоростных видеокамер, что объясняет большое число выделенных режимов течения. Более достоверным является подход, основанный на анализе сигналов от лазерных измерительных систем, примененный для определения характеристик кипения хладона $\mathrm{R}-134 \mathrm{a}[8]$ и течения смеси азот-вода [9] в микроканалах при Во > 0.1. Работы по определению структурных характеристик газожидкостного течения в условиях доминирующего влияния капиллярных сил (Во $<<1)$, полученных с использованием лазерного сканирования, в литературе не представлены. Вместе с тем достоверное установление границ режимов течения в этих условиях и определение их свойств критически важны для разработки микрожидкостных систем.

Целью настоящей работы является комплексное исследование структурных характеристик течения смеси азот-вода в горизонтальном микроканале в условиях доминирующего влияния капиллярных сил $($ Во $=0.012)$. Структурные характеристики течения впервые установлены с использованием двухлучевого лазерного сканирования и скоростной видеосъемки на расстоянии 500 калибров от входа в широком

Письма в ЖТФ, 2017, том 43, вып. 21 
диапазоне приведенных скоростей газа и жидкости. На основе статистического анализа данных лазерного сканирования предложен новый метод идентификации режимов течения и определены их свойства.

Эксперименты проведены с микроканалом прямоугольного сечения размером $250 \times 315 \mu \mathrm{m}$ и длиной $300 \mathrm{~mm}$, две стенки которого выполнены из оптического стекла, что обеспечивает возможность лазерного сканирования течения. Описание экспериментальной установки приведено в [10]. При проведении экспериментов газ и жидкость подаются в боковые плечи Т-образного смесителя и далее газожидкостный поток направляется в микроканал. Регулировка расходов жидкости и газа осуществляется посредством термомассовых контроллеров расхода фирмы Bronkhorst с точностью измерения расходов газа $0.12 \mathrm{n} . \mathrm{ml} / \mathrm{min}$ и жидкости $0.002 \mathrm{~g} / \mathrm{s}$. На выходе из экспериментального участка газожидкостная смесь разделяется, и вода откачивается перистальтическим насосом в открытый бак. На входе в канал расположен отборник датчика статического давления.

Для исследования режимов газожидкостного течения использован метод двулучевого лазерного сканирования. Два лазера с мощностью $1 \mathrm{~mW}$ располагались так, что лазерные лучи через линзы освещали длинную сторону прямоугольного канала на расстоянии $53 \mathrm{~mm}$ один от другого. Первый лазер расположен на расстоянии $125 \mathrm{~mm}$ от входа. Интенсивность лазерного излучения, прошедшего через канал с газожидкостной средой, измерялась фотодиодами с апертурой $200 \mu \mathrm{m}$, расположенными на противоположной стороне канала. Сигналы с фотодиодов регистрировались с помощью высокоскоростной платы аналого-цифрового преобразователя (Lcard L 264) и обрабатывались на компьютере. Объемный расход газа определялся по заданному массовому расходу и статическому давлению в месте расположения фотодиодов.

С лицевой стороны канала проводилась визуализация течения при помощи цифровой видеокамеры AOS X-Pri.

Применение двухлучевого лазерного сканирования, совмещенного со скоростной видеосъемкой, позволило достоверно определить режимы течения и построить взаимно корреляционные функции, характеризующие степень корреляции сигналов с фотодиодов. Наблюдаемые режимы течения были классифицированы следующим образом.

1. Периодическое течение с удлиненными пузырями-снарядами $(P)$ наблюдается в диапазоне приведенных скоростей газа от 0.08 до $0.6 \mathrm{~m} / \mathrm{s}$.

Письма в ЖТФ, 2017, том 43, вып. 21 


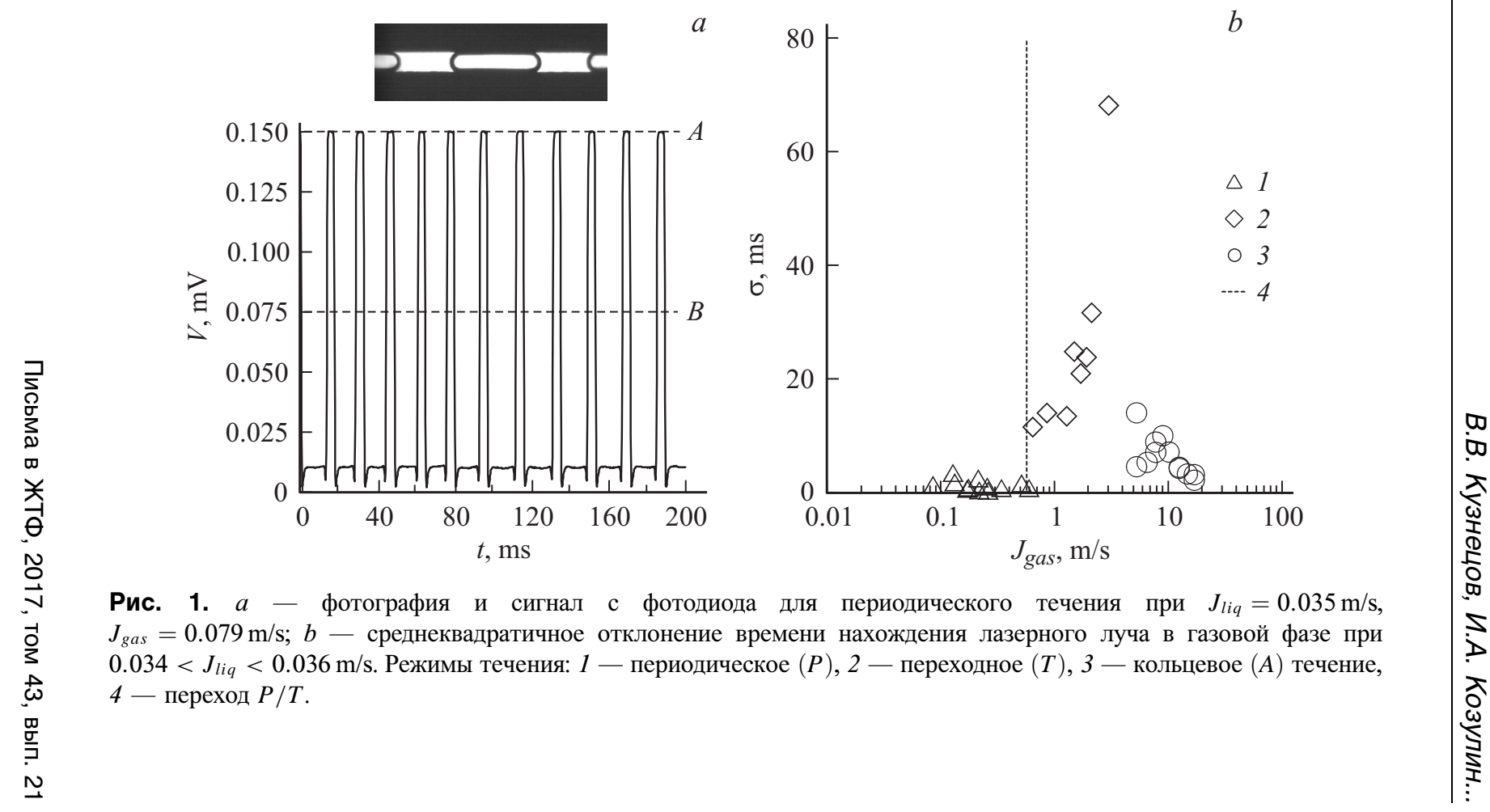


Вид периодического течения и фрагмент сигнала с первого фотодиода, который характеризует структурные характеристики течения, показаны на рис. $1, a$. Максимальный уровень напряжения (линия $A$ ) соответствует полному заполнению канала жидкостью (жидкая пробка), минимальный уровень напряжения соответствует заполнению канала газовой фазой с пленкой жидкости на стенках (удлиненный пузырь-снаряд). Для определения распределения времени нахождения лазерного луча в газовой фазе с учетом наличия не только жидких пробок, но и волн возмущения на поверхности пленки был выбран уровень напряжения, показанный штриховой линией $B$ на рис. $1, a$. Полагалось, что уровень напряжения ниже линии $B$ соответствует прохождению луча через газовую фазу. На рис. $1, b$ представлено среднеквадратичное отклонение распределения времени нахождения лазерного луча в газовой фазе в зависимости от величины приведенной скорости газа. Экспериментальные данные соответствуют фиксированной приведенной скорости жидкости в диапазоне от 0.034 до $0.036 \mathrm{~m} / \mathrm{s}$. Как видно, при приведенной скорости газа $0.59 \mathrm{~m} / \mathrm{s}$, значение среднеквадратичного отклонения сигнала резко возрастает. Это показывает нарушение периодичности следования пузырей-снарядов и возникновение переходного течения.

2. Переходное течение $(T)$, представленное перемежающимся течением удлиненных пузырей-снарядов и кольцевым течением, наблюдается в диапазоне приведенных скоростей газа от 0.6 и до $4 \mathrm{~m} / \mathrm{s}$. Фрагмент сигнала с первого фотодиода и вид переходного течения показаны на рис. 2, $a$. Как видно, при переходном течении дополнительно к жидким пробкам наблюдаются волны возмущения на поверхности жидкости, амплитуда которых меньше уровня сигнала для жидких пробок. Такое течение нестабильно, жидкие пробки возникают при объединении волн возмущений на противоположных сторонах канала для кольцевого течения и разрушаются при объединении газовых пузырей-снарядов. Его особенностью является изменяющаяся во времени амплитуда сигнала, среднее значение которой существенно меньше для кольцевого течения, чем для течения пузырей-снарядов (рис. $1, a$ ). На рис. $2, b$ представлено время нахождения лазерного луча в газовой фазе (светлые точки) и жидкой фазе (темные точки), отнесенное к интервалу измерения $T_{r e l}$, в зависимости от приведенной скорости газа. Измерения проведены при фиксированной приведенной скорости жидкости в диапазоне от 0.034 до $0.036 \mathrm{~m} / \mathrm{s}$. На этом рисунке для определения наличия жидких пробок данные представлены для уровня сигнала, равного 0.9 от уровня 

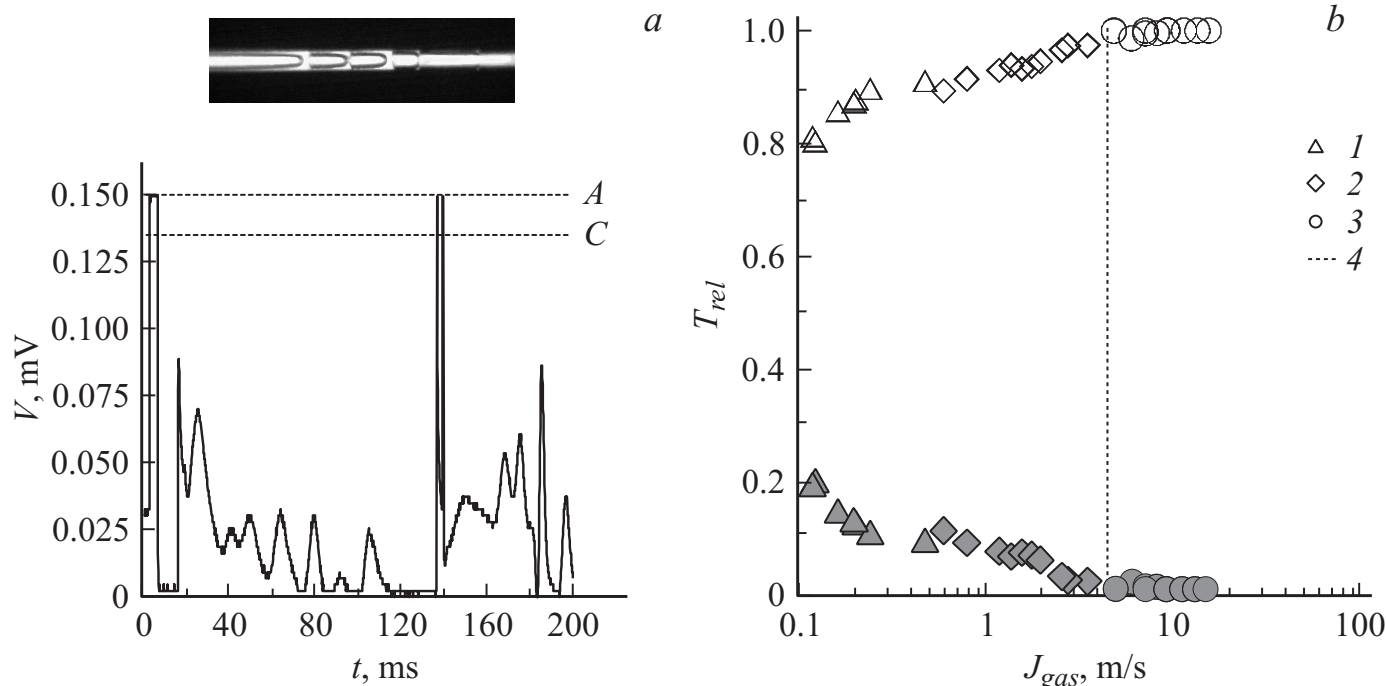

Pис. 2. $a$ - фотография и сигнал с фотодиода для переходного течения при $J_{l i q}=0.035 \mathrm{~m} / \mathrm{s}$ и $J_{\text {gas }}=9.31 \mathrm{~m} / \mathrm{s}$; $b-$ относительное время нахождения лазерного луча в газовой и жидкой фазах $T_{r e l}$ при $0.034<J_{\text {liq }}<0.036 \mathrm{~m} / \mathrm{s}$. Режимы течения обозначены на рис. $1, b ; 4-$ переход $T / A$. Светлые точки - газ, темные - жидкость. 


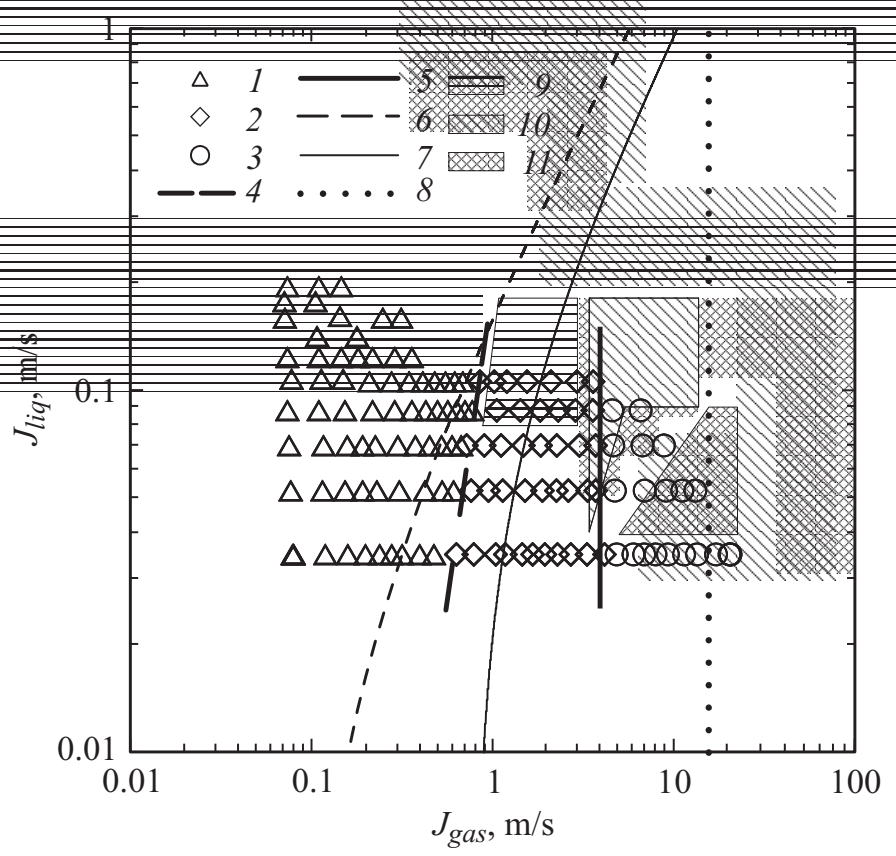

Рис. 3. Карта режимов течения в микроканале.

сигнала, соответствующего полному заполнению канала жидкостью (пунктирная линия $C$ на рис. $2, a$ ). Как видно, при приведенной скорости больше $4 \mathrm{~m} / \mathrm{s}$ жидкие пробки в микроканале исчезают, и относительное время нахождения лазерного луча в газовой фазе $T_{r e l}$ достигает единицы. В этом случае наблюдается переход в кольцевому течению с непрерывным газовым ядром. Следует отметить, что относительное время существования жидких пробок для переходного течения на рис. $2, b$ монотонно уменьшается с ростом приведенной скорости газа.

3. Кольцевое течение $(A)$, при котором газовое ядро непрерывно и пленка жидкости с волнами возмущения движется по стенкам канала, наблюдается при приведенной скорости газа больше $4 \mathrm{~m} / \mathrm{s}$. Его особенностью является существенно меньшая амплитуда сигнала, чем для течения с удлиненными пузырями, из-за отсутствия жидких пробок. При увеличении приведенной скорости газа амплитуда волн

Письма в ЖТФ, 2017, том 43, вып. 21 
возмущений и среднеквадратичное отклонение распределения времени нахождения лазерного луча в газовой фазе уменьшаются (рис. $1, b$ ), и для скорости $11 \mathrm{~m} / \mathrm{s}$ такие волны практически исчезают. Для большей приведенной скорости газа на поверхности жидкости наблюдаются только волны ряби малой амплитуды.

Полная карта режимов течения в координатах приведенная скорость жидкости-приведенная скорость газа показана на рис. 3. Здесь точками 1 показаны области периодического течения с удлиненными пузырями-снарядами $(P)$, точками 2 - переходного режима $(T)$, точками 3 - кольцевого течения, полученные при визуальном анализе сигналов с фотодиодов. Штриховой линией 4 показана граница периодическое/переходное течение, определенная по скорости газа, при которой резко возрастает среднеквадратичное отклонение, линией 5 представлена граница переходное/кольцевое течение, определенная по скорости газа, при которой относительное время $\tau$ нахождения лазерного луча в газовой фазе достигает единицы (при фиксированной скорости жидкости). Линиями 6-8 показаны соответственно границы режимов течения снарядное/вспененное, вспененное/кольцевое, кольцевое/дисперсное, определенные по уравнениям из работы [11] для обычных каналов. Заштрихованные области на этом рисунке показывают режимы течения, наблюдаемые в работе [7] для микроканалов прямоугольного сечения с Т-образным входом: 9 - нестабильное снарядное, 10 - снарядно-кольцевое и 11 - кольцевое течение. Как видно, выделенные с использованием лазерного сканирования области характерных режимов течения в целом соответствуют наблюдаемым ранее, но значительно отличаются от режимов течения в обычных каналах.

В заключение отметим, что измерение структурных характеристик газожидкостного течения с использованием двухлучевого лазерного сканирования и их статистическая обработка позволили установить границы режимов течения в микроканале в условиях доминирующего влияния капиллярных сил и определить их свойства. Предложенный метод идентификации режима течения является перспективным и будет применен для течений с существенно другими физическими свойствами жидкости и газа для построения обобщенных карт режимов течений.

Исследование выполнено в ИТ СО РАН за счет гранта Российского фонда фундаментальных исследований (проект № 15-08-07506 А).

Письма в ЖТФ, 2017, том 43, вып. 21 


\section{Список литературы}

[1] Abramson A.R., Tien C.L. // J. Microscale Thermophys. Eng. 1999. V. 3. N 4. P. 229-244.

[2] Rebrov E.V. // Theor. Found. Chem. Eng. 2010. V. 44. P. 355-367.

[3] Chung P.M.Y., Kawaji M., Kawahara A., Shibata Y. // J. Fluids Eng. 2004. V. 126. P. 546-552.

[4] Choi C.W., Yu D.I., Kim M.H. // Int. J. Heat Mass Transfer. 2011. V. 54. P. 616 624.

[5] Чиннов Е.А., Роньшин Ф.В., Кабов О.А. // Письма в ЖТФ. 2015. Т. 41. В. 17. C. $1-8$.

[6] Waelchli S., von Rohr P.R. // Int. J. Multiphase Flow. 2006. V. 32. P. 791-806.

[7] Zhao Y., Chen G., Ye C., Yua Q. // Chem. Eng. Sci. 2013. V. 87. P. 122-132.

[8] Revellin R., Dupont V., Ursenbacher T., Thome J.R., Zun I. // Int. J. Multiphase Flow. 2006. V. 32. P. 755-774.

[9] Kozulin I.A., Kuznetsov V.V. // J. Appl. Mech. Tech. Phys. 2011. V. 52. P. 956964.

[10] Kuznetsov V.V., Shamirzaev A.S., Kozulin I.A. // J. Phys.: Conf. Ser. 2012. V. 395. Iss.1. P. 012093.

[11] Mishima K., Ishii M. // Int. J. Heat Mass Transfer. 1984. V. 27. P. 723-737.

Письма в ЖТФ, 2017, том 43, вып. 21 